\title{
Clinical, biochemical and histological characteristics of a Sri Lankan population of non-alcoholic steatohepatitis (NASH) patients
}

\section{SJ De S Hewavisenthi ${ }^{1}$, AS Dassanayaka ${ }^{2}$ and HJ De Silva ${ }^{3}$}

(Index words : Diabetes, high fat intake, hyperlipidaemia, management obesity)

\begin{abstract}
Background Non-alcoholic steatohepatitis (NASH) is common and can progress to cirrhosis. It has been regarded as a 'disease of affluence' and there are only a few reports from developing countries.

Objective To describe the clinical, biochemical, and histological characteristics of a cohort of NASH patients in Sri Lanka, and to determine their short term outcome following modifications of lifestyle.

Method Patients who had a liver biopsy for investigation of raised hepatic enzymes were assessed during the period May 1999 - May 2003. Patients who had an alcohol intake of over $40 \mathrm{~g} /$ week were excluded. Detailed clinical and biochemical data of patients with histologically confirmed $\mathrm{NASH}$ were compiled. Histological grading and staging was done using the Brunt system. The patients were advised on lifestyle modifications and the control of diseases known to be associated with NASH. They were followed up at 3 -monthly intervals.
\end{abstract}

Results During the study period liver biopsies were performed on 296 patients and 100 (35.1\%) were diagnosed as having NASH. (Men $=79$, Mean age 37.2 years, SD 10.6). Risk factors for NASH included diabetes mellitus (55\%), obesity (52\%), hyperlipidaemia (54\%), a family history of risk factors $(66 \%)$ and a high dietary fat intake (66\%). However, $44.3 \%$ of men and $33.3 \%$ of women were not overweight. The histological grading and staging of 80 biopsies showed Grade 1 in $31(38.8 \%)$, Grade 2 in $29(36.3 \%)$, Grade 3 in $20(25 \%)$, Stage 1 in $57(71.3 \%)$ Stage 2 in $13(16.3 \%)$, Stage 3 in $2(2.5 \%)$ and Stage 4 in 8 (10\%). In 55/91 (60.4\%) patients who were followed up for a median of 2.5 years (range 1-4 years) the serum transaminases returned to normal in a median of 7 months (range 3-14 months).

Conclusion The clinical, biochemical and histological features of NASH patients in our series are similar to that reported in western countries. However "lean males" accounted for a significant proportion. In the short term a majority of patients showed improvement in serum transaminases with lifestyle modification.

\section{Introduction}

Over the last 20 years non-alcoholic steatohepatitis (NASH) has increasingly been recognised as a common chronic liver disease. The clinical significance of NASH is two-fold: firstly, the potential for mortality, due to progression to cirrhosis and complications of portal hypertension, hepatic insufficiency and rarely, hepatocellular carcinoma [1-4]; secondly, the frequency with which it is encountered in patients who undergo evaluation for abnormalities in liver enzymes [5-7].

NASH is widely recognised as the second most important cause of liver disease in the USA and several other developed countries such as Japan and Australia [7]. Risk factors for its development include diabetes, obesity, and a high fat intake, all common in rich countries. Many developing countries in the Asian region have factors that predispose to the development of NASH [8].

Senior lecturers, Department of Pathology ${ }^{1}$ and Department of Pharmacology ${ }^{2}$, and Professor in Medicine ${ }^{3}$, Faculty of Medicine, University of Kelaniya, Sri Lanka. Correspondence SJDeSH, e-mail: <prajafer@mail.ewisl.net> Received 20 January 2005, and accepted 2 April 2005. Competing interests: none declared. 
For example, by the year $2020,60 \%$ of all patients in the world with diabetes mellitus are projected to live in Asia [9]. Data on NASH from the developing countries, including those in the Asia-Pacific region are sparse [10].

The objective of our study was to describe and characterise the clinical, biochemical and histological features of a group of Sri Lankan patients with NASH and to assess the short term clinical outcome following lifestyle modifications.

\section{Methods}

During a 4-year period from May1999 to may 2003, liver biopsies were performed on 296 patients who had elevated serum transaminase levels for more than 6 months. Hundred of them were diagnosed as having NASH. The histological criteria for diagnosis of NASH included macrovacuolar steatosis of hepatocytes, lobular inflammation including scattered polymorphs and mononuclear cells, hepatocyte ballooning, and perisinusoidal, perivenular or pericellular fibrosis [11]. Clinical and biochemical details were recorded at the first visit. The clinical details included a history of diabetes, hyperlipidaemia, drug treatment, an assessment of alcohol and dietary fat intake, and exercise. A family history of co-morbid factors associated with NASH was also assessed. The height and weight were recorded and body mass index calculated. In addition to liver transaminases, the following investigations were performed: lipid profile, a fasting and post-prandial blood glucose (venous), serum insulin, hepatitis viral serology, alpha 1 antitrypsin, caeruloplasmin level, anti-nuclear antibody, anti-smooth muscle antibody, serum iron and total iron binding capacity, and ESR.

Patients with NASH were advised on a low fat diet by a nutritionist, regular exercise, and treated for diabetes and hyperlipidaemia where appropriate. They were followed up at 3-month intervals. Their weight and serum transaminase levels were assessed every 3 months.

\section{Results}

In $65 / 100$ patients, elevated serum transaminase levels were incidental findings detected at routine medical examinations (patients were asymptomatic). Fifteen had right hypochondrial pain, 11 had an enlarged liver, and 9 had been investigated for chronic fatigue.

Table 1 shows the associated risk factors for NASH in the 100 patients. Some of the patients had more than one risk factor. However, 35/79 males and 7/21 females were not overweight.

Table 2 shows the details of biochemical investigations. Gamma glutamyl transferase was raised in $50 \%$ of patients and serum bilirubin was slightly raised in $15 \%$. Serum iron and total iron binding capacity were abnormal in $12 \%$ and $20 \%$. Other investigations were normal.

The histological features of liver biopsies in 80 patients is shown in Table 3. Grading and staging based on some of these histological features, done according to the system proposed by Brunt [11], show that 31/80 (38.8\%) were Grade 1 (mild), 29/80 (36.3\%) Grade 2, and 20/80 (25\%) Grade 3 (severe), and 57 (71.3\%) were Stage 1, $13(16.3 \%)$ Stage 2, 2 (2.5\%) Stage 3, and 8 (10\%) Stage 4.

Table 1. Risk factors for NASH $(\mathbf{n}=100)$

\begin{tabular}{ll}
\hline Risk factor & Number of patients (\%) \\
\hline Diabetes & 55 \\
Obesity & 52 (if BMI >23 is used 67\%) \\
Hyperlipidaemia & 54 \\
Family History & 66 \\
$\quad$ Atherosclerosis & 40 \\
$\quad$ Liver disease & 14 \\
$\quad$ Hyperlipidaemia & 13 \\
$\quad$ Diabetes mellitus & 55 \\
High fat intake & 66 \\
\hline
\end{tabular}

Table 2. Abnormal biochemical indices $(n=100)$

\begin{tabular}{lc}
\hline Abnormal index & Number of patients $(\%)$ \\
\hline Serum transaminases elevated & 100 \\
AST/ALT ratio < 1 & 90 \\
Serum bilirubin elevated & 15 \\
Gamma glutamyl & 50 \\
$\quad$ transferase elevated & 5 \\
Alkaline phosphatase elevated & 59 \\
Lipid pattern abnormal & \\
Fasting blood glucose $*$ \} & 18 \\
Post-prandial blood glucose $*$ & \\
\hline
\end{tabular}

* Patients with biochemical abnormalities only. Most diabetic patients on treatment had normal indices.

Table 3. Histological features of the liver biopsies of NASH patients $(n=80)$

\begin{tabular}{lcccc}
\hline Feature & $\begin{array}{c}\text { Absent } \\
(\%)\end{array}$ & $\begin{array}{c}\text { Mild } \\
(\%)\end{array}$ & $\begin{array}{c}\text { Moderate } \\
(\%)\end{array}$ & $\begin{array}{c}\text { Severe } \\
(\%)\end{array}$ \\
\hline $\begin{array}{l}\text { Steatosis } \\
\text { Ballooning of } \\
\text { hepatocytes }\end{array}$ & $0(0)$ & $17(21.3)$ & $32(40.0)$ & $31(38.8)$ \\
$\begin{array}{l}\text { Lobular } \\
\text { inflammation }\end{array}$ & $0(0)$ & $34(42.5)$ & $36(45)$ & $10(12.5)$ \\
$\begin{array}{l}\text { Portal } \\
\text { inflammation }\end{array}$ & $3(3.8)$ & $32(40.5)$ & $38(48.5)$ & $7(8.9)$ \\
$\begin{array}{l}\text { Mallory's } \\
\text { hyaline }\end{array}$ & $27(29.7)$ & $32(40.5)$ & $17(21.5)$ & $4(5.1)$ \\
$\begin{array}{l}\text { Lipogranuloma } \\
\text { 3ina }\end{array}$ & $31(38.8)$ & $29(36.5)$ & $20(36.3)$ & $0(0)$ \\
\hline
\end{tabular}

Patients were followed up for a median of 2.5 years (range 1-4 years). Nine patients did not attend a single follow up visit. Serum transaminase levels fell to the normal range in 27/91 (29.7\%) patients at 3-6 months, $24 / 91$ (26.4\%) at 6-12 months, and over 1 year in 4/91 (4.3\%). In $36 / 91(39.6 \%)$ patients the serum transaminase levels had 
not normalised at the last follow up visit. During follow up 7/91 $(7.7 \%)$ patients had a repeat elevation of transaminase levels after initial normalisation. They all admitted to neglecting the advise on lifestyle modifications.

\section{Discussion}

In other series of patients undergoing liver biopsy, the prevalence of NASH has been found to be $15-34 \%$ [12-14]. This wide range in prevalence is probably related to differences in study design. The prevalence of NASH in our series of patients undergoing liver biopsy for unexplained elevation of liver enzymes was $35.1 \%$. Because patients undergoing liver biopsy are highly selected, this data will not reflect the true prevalence of NASH or non-alcoholic fatty liver disease (NAFLD) in the general population.

The age at presentation of patients was low compared to previous series $[1,4,10,15]$. Our series included two children aged 12 and 14 . We observed a lower proportion of obese women among our series, and $44.3 \%$ of men were neither obese nor overweight. Other series have reported men with NASH who were of normal weight, without diabetes and hyperlipidaemia $[15,16]$. In one, more than $50 \%$ of patients were non-obese males without diabetes or hypertriglyceridemia [15]. More than half of those patients had elevated iron indices and some had increased stainable iron in liver biopsies. The investigators have suggested that these patients might be heterozygous for hereditary haemochromatosis. Although some of our patients had mildly abnormal iron indices, none had an increase in stainable iron in the liver biopsies. The number of obese patients in our series would have increased if the cut-off BMI for assessing obesity is reduced to 23, which is considered to be more appropriate for Asian populations than 25 that is used in western studies [17].

Most of the patients in our series $(65 \%)$ were asymptomatic and elevated liver enzymes were an incidental finding at routine medical examinations. This is in keeping with several other studies where $45-100 \%$ of patients were asymptomatic $[18,19]$. Serum transaminase levels were elevated in all patients, mainly a to 2-5-fold increase. Only two patients had a 10-fold increase. The AST/ALT ratio was less than one in $90 \%$ of patients, and this is also in keeping with the reported frequency of $65-90 \%$. A ratio of more than one suggests more advanced disease, which was seen in 10 patients. These patients had higher Stages (3 and 4 ) of fibrosis on Brunt staging. The histological patterns showed a preponderance of Grades 1 and 2. Fibrosis was found in all patients, as pericellular, perisinusoidal or perivenular fibrosis was a criterion for diagnosis of NASH. More advanced stages of fibrosis were found in only $23 / 80$ patients, and established cirrhosis in only $8(10 \%)$.

The prognosis in NAFLD and NASH seems to be determined mainly by the severity of histological liver damage. Some patients with NAFLD follow a relatively benign course, whereas in others, the disease may progress to cirrhosis and its complications [2,15,18-22]. We did not perform repeat liver biopsies. However, $39.6 \%$ of the patients did not show significant improvement in their serum transaminase levels even after lifestyle modifications. Patients with NASH show demographic features similar to those previously reported, but we found a significant proportion of men who are non-obese, who do not fit into the typical picture for NAFLD or NASH. The majority of patients $(60.4 \%)$ appear to have benefited from lifestyle modifications in the short term, with improvement in biochemical liver transaminase tests.

\section{References}

1. Diehl AM. Nonalcoholic steatohepatitis. Seminars in Liver Disease 1999;19:221-9.

2. Powell EE, Cooksley WGE, Hanson R, Searle J, Halliday JW, et al. The natural history of nonalcoholic steatohepatitis: a follow up study of forty two patients for up to 21 years. Hepatology 1990;11: 74-80.

3. Lonardo A. Fatty liver and nonalcoholic steatohepatitis. Where do we stand and where are we going? Digestive Diseases 1999:17: 80-9.

4. Ludwig J, McGill DB, Lindor KD. Metabolic liver disease review: nonalcoholic steatohepatitis. Journal of Gastroenterology and Hepatology 1997;12: 398-403.

5. Baldridge AD, Perez-atayde AR, Graeme-Cook F, Higgins L, Lavine JE. Idiopathic steatohepatitis in childhood: a multicenter study. Journal of Paediatrics 1995;127: 700-4.

6. Byron D, Minuk GY. Clinical hepatology: Profile of an urban, hospital-based practice. Hepatology 1996; 24: 813-5.

7. James O, Day C. Non-alcoholic steatohepatitis: another disease of affluence. [Commentary] Lancet 1999; 353: 1634-6.

8. Basnyat B, Rajapaksa LC. Cardiovascular and infectious disease in South Asia: the double whammy. British Medical Journal 2004; 328: 781.

9. Zimmet P, Alberti KG, Shaw J. Global and societal implications of the diabetes epidemic. Nature 2001; 414: 782-7.

10. Chitturi S, Farrell GC, George J. Non-alcoholic steatohepatitis in the Asia-Pacific region: Future shock? Journal of Gastroenterology and Hepatology 2004; 19: 368-74.

11. Brunt EM, Janney CG, Di Bisceglie AM, NeuschwanderTetri BA, Bacon BR. Nonalcoholic steatohepatitis: a proposal for grading and staging the histological lesions. American Journal of Gastroenterology 1999; 94: 2467-2474.

12. Nonomura A, Mizukami Y, Unoura M, Kobayashi K, Takeda Y, et. al. Clinicopathological study of alcohol-like liver disease in non-alcoholics; non-alcoholic steatohepatitis and fibrosis. Gastroenterologica Japonica 1992; 27 : 521-8.

13. Propst A, Propst T, Judmaier G, Vogel VW. Prognosis in nonalcoholic steatohepatitis. [Letter] Gastroenterology 1995; 108: 1607.

14. Hulcrantz R, Glaumann H, Lindberg G, Nilsson LH. Liver investigation in 149 asymptomatic patients with 


\section{Papers}

moderately elevated activities of serum aminotransferases. Scandinavian Journal of Gastroenterology 1986;21:109-13.

15. Bacon BR, Farahvash MJ, Janney CG, NeuschwanderTetri BA. Nonalcoholic steatohepatitis: an expanded clinical entity. Gastroenterology 1994; 107: 1103-9.

16. Agarwal SR. Malhotra V, Sakhuja P, Sarin SK. Clinical, biochemical and histological profile of nonalcoholic steatohepatitis. Indian Journal of Gastroenterology 2001; 20: $183-6$.

17. Farrell GC. Non-alcoholic steatohepatitis: what is it, and why is it important in the Asia-Pacific region? Journal of Gasteroenterology and Hepatology 2003; 18: 124-38.

18. Lee RG. Nonalcoholic steatohepatitis. A study of 49 patients. Human Pathology 1989; 20: 594-8.
19. Teli MR, James OFW, Burt AD, Bennett MK, Day CP, The natural history of nonalcoholic fatty liver. A follow up study. Hepatology 1995; 22: 1714-9.

20. Pinto HC, Baptista A, Camilo ME, Valente A, Saragoca A, Carniero de Maura M. Non-alcoholic steatohepatitis. Clinicopathological comparison with alcoholic hepatitis in ambulatory and hospitalized patients. Digestive Diseases and Sciences 1996; 41: 172-9.

21. Andersen T, Gluud C, Franzmann MD, Christoffersen P. Hepatic effects of dietary weight loss in morbidly obese subjects. Journal of Hepatology 1991; 12: 224-9.

22. Falck-Ytter Y, Younossi ZM, Marchesini G, McCullough AJ. Clinical features and natural history of nonalcoholic steatosis syndromes. Seminars in Liver Disease 2001; 21: $17-26$. 